\title{
Microstructural Characterization of CdTe and CIGS Thin Film Solar Cells by Analytical Transmission Electron Microscopy
}

\author{
U. Sharma \& D. Susnitzky
}

Evans Analytical Group, Sunnyvale, CA, 94085

In contrast to thin film $\mathrm{Si}, \mathrm{CdTe}$ and $\mathrm{CuIn}_{\mathrm{x}} \mathrm{Ga}_{1-\mathrm{x}} \mathrm{Se}_{2}$ (CIGS) solar cells have multiple layers that, by design, are not planar. These materials include very thin $(<50 \mathrm{~nm})$ layers concomitant with thicker (1$5 \mu \mathrm{m})$ layers. The rough surface topography, combined with a large range of layer thicknesses can compromise the spatial resolution of analytical techniques based on depth profiling. We have found that TEM combined with Energy Dispersive X-ray Spectroscopy (EDS) is ideally suited for microstructural characterization and local elemental analysis of these photovoltaic materials.

For this study, TEM cross section samples were prepared by the in-situ lift out technique using an Omniprobe Autoprobe fitted to an FEI Altura 835 focused ion beam (FIB) tool. TEM imaging and microanalysis were performed on an FEI Tecnai TF-20XT FEGTEM fitted with a $30 \mathrm{~mm}^{2}$ Oxford INCA EDS detector and a Fischione HAADF detector.

Fig. 1 shows three bright field (BF) TEM images from CdTe thin film stacks. Low magnification image (1a) presents the entire thin film stack $(\sim 2.5 \mu \mathrm{m})$ while higher magnification images $(1 \mathrm{~b}, 1 \mathrm{c})$ allow precise measurements of the thinner films. Grain boundaries and other internal defects like stacking faults, twins and dislocations are seen in these BF images due to diffraction contrast effects.

Fig. 2 shows HAADF-STEM or 'Z-contrast' images of a) CIGS stack b) CdS buffer and ZnO contact c) multiphase region in one area of CIGS. The deposition of CIGS can be performed in various ways for e.g., co-evaporation or precursor/reaction and in single or multiple steps [1]. These different approaches might lead to local variations in composition, intentional or otherwise. Vertical EDS linescan in 'd' shows falling Ga and rising In levels from bottom to top of CIGS layer. Note that Ga levels implanted into CIGS during FIB preparation are too low to be detected by EDS as evidenced from lack of Ga detected at the top of the CIGS layer. Multiphase regions in ' $c$ ' show different relative levels of $\mathrm{Cu}, \mathrm{In}, \mathrm{Ga}$, Se and $\mathrm{O}$ as shown in comparative spectra in 'e'.

Fig. 3 a) is an HAADF STEM image of a CdS/CdTe interface. Small regions of mottled contrast were observed in both $\mathrm{CdS}$ and $\mathrm{CdTe}$ grains that were different from the rest of the film structure. EDS scans along the grain boundary and interior of CdTe grain show $\mathrm{Cl}$ at the $\mathrm{CdS} / \mathrm{CdTe}$ interface and higher levels of $\mathrm{S}$ at the CdTe grain boundary when compared to similar depths inside the CdTe grain. Presence of $\mathrm{Cl}$ likely indicates post-deposition $\mathrm{CdCl}_{2}$ treatment for $\mathrm{CdTe}$ [2].

With the examples above, we have demonstrated that analytical TEM is a powerful technique to characterize the microstructure and composition of CdTe and CIGS based thin film photovoltaics.

References

[1] B. M. Keyes et al., NREL/CP-520-31427, May 2002

[2] R.W. Birkmire et al., Proc. 24 ${ }^{\text {th }}$ IEEE Photovoltaic Specialist Conf., Dec 1994 v.1, pp 76-82

[3] The authors gratefully acknowledge discussions with Dr. Richard Hockett and Dr. Larry Wang 

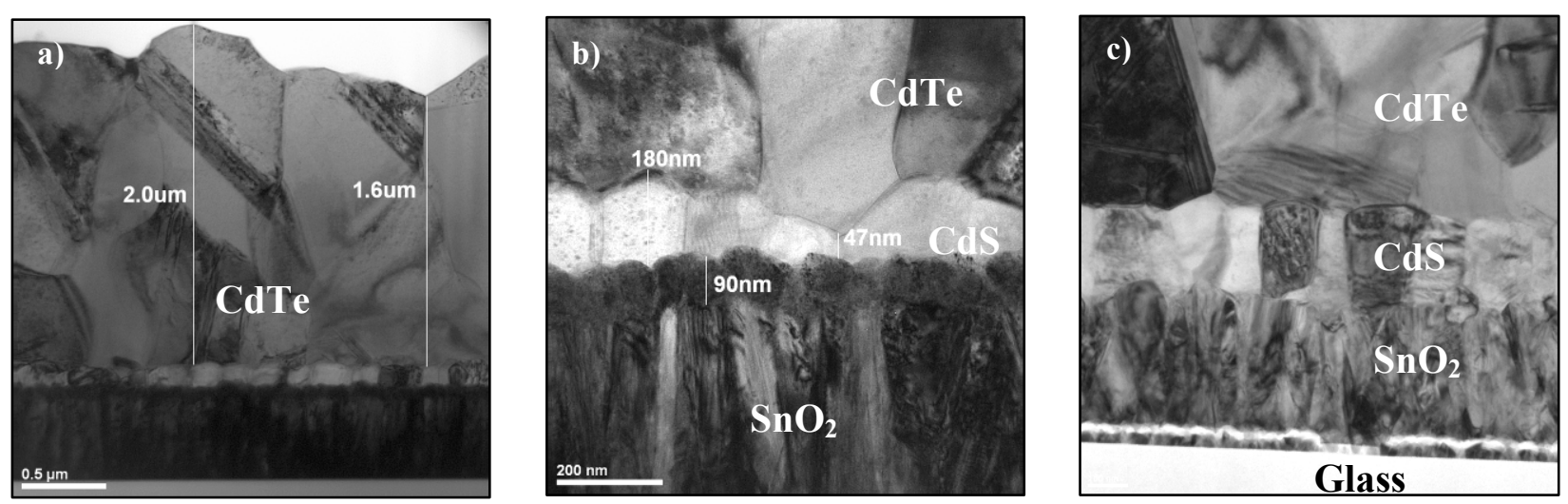

Fig. 1 Bright field TEM images of CdTe thin film solar cells. Grain boundaries and internal defects within the CdTe and barrier layers are readily observed.
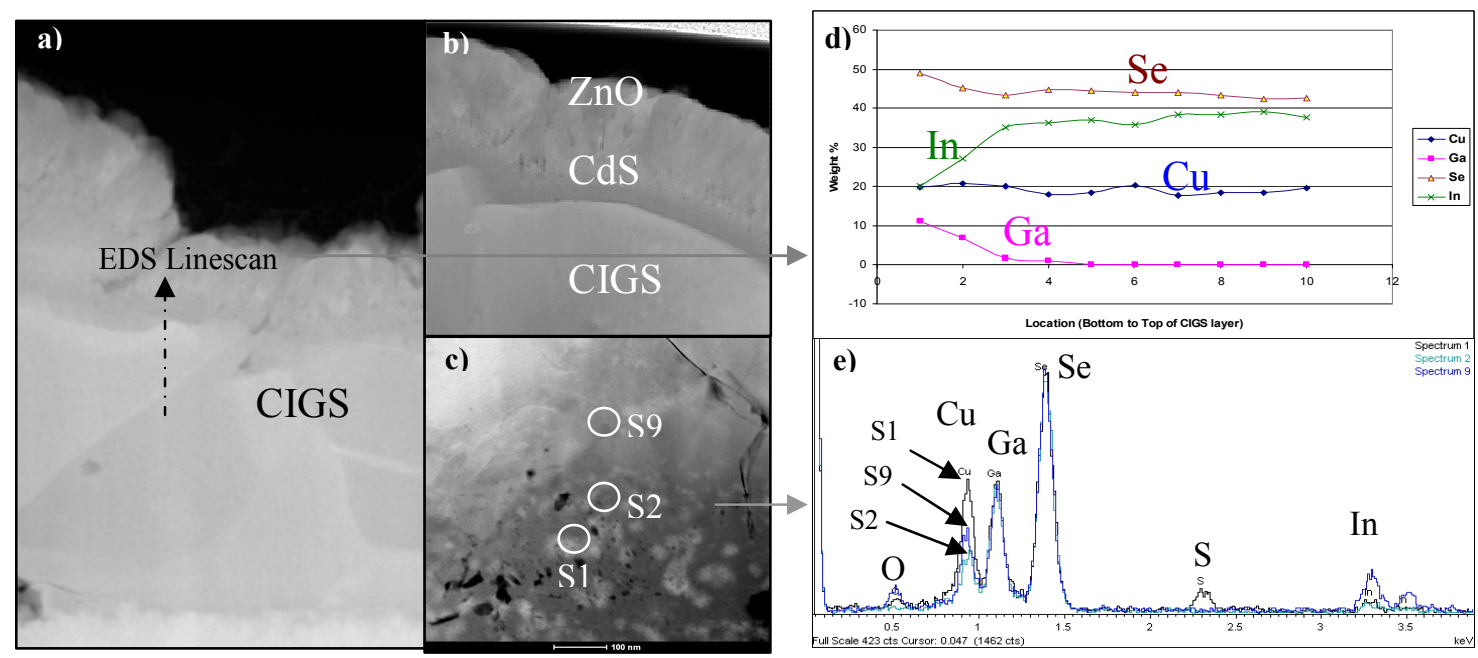

Fig. 2 HAADF-STEM images of a) CIGS solar cell showing location of EDS linescan; b) Top $\mathrm{CdS} / \mathrm{ZnO}$ layers; c) multiphase regions; d) Element profiles from 'a' showing Ga and In gradients and e) Spot $\mathrm{S} 1$ shows $\mathrm{S}$ and relatively higher $\mathrm{Cu}$ compared to $\mathrm{S} 2$ that shows much lower $\mathrm{Cu}$ and no $\mathrm{S}$. Spot $\mathrm{S} 9$ shows higher In and some $\mathrm{O}$ in addition to $\mathrm{Cu}, \mathrm{Ga}$ and $\mathrm{Se}$.

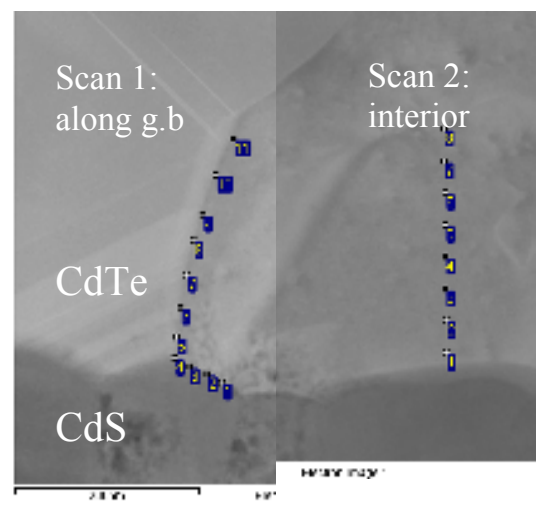

Table 1: EDS scan along phase boundary and grain boundary

\begin{tabular}{c|llll}
\hline Spectrum & $\mathrm{S}$ & $\mathrm{Cl}$ & $\mathrm{Cd}$ & $\mathrm{Te}$ \\
1 & 29.3 & 4.9 & 42.4 & 23.4 \\
2 & 16.1 & 4.8 & 35.0 & 44.1 \\
3 & 24.4 & 4.0 & 45.4 & 26.2 \\
4 & 17.1 & 5.1 & 44.7 & 33.2 \\
5 & 3.2 & & 47.7 & 49.1 \\
6 & 2.9 & & 50.7 & 46.4 \\
7 & 2.1 & & 48.2 & 49.7 \\
8 & 2.1 & & 48.4 & 49.5 \\
9 & 2.2 & & 47.4 & 50.4 \\
10 & 2.0 & & 49.2 & 48.7 \\
11 & 2.7 & & 50.8 & 46.5 \\
\hline
\end{tabular}

Table 2: EDS scan inside grain

\begin{tabular}{l|lll}
\hline Sp. & S & Cd & Te \\
1 & 4.4 & 49.4 & 46.2 \\
2 & 3.2 & 49.5 & 47.3 \\
3 & 2.1 & 49.7 & 48.2 \\
4 & 3.0 & 49.7 & 47.3 \\
5 & 2.3 & 49.8 & 47.8 \\
6 & & 51.5 & 48.5 \\
7 & & 48.8 & 51.2 \\
8 & & 48.7 & 51.3 \\
\hline \multicolumn{4}{l}{ All results in atomic\% }
\end{tabular}

Fig. 3 a) HAADF-STEM image of CdS/CdTe layers showing location of EDS scan along the grain boundary and inside of grain respectively. $\mathrm{Cl}$ was seen in the $\mathrm{CdS} / \mathrm{CdTe}$ interface and higher levels of $\mathrm{S}$ were observed along the CdTe grain boundary compared to the interior. 